\title{
Late Complications of Haemodialysis Access: Appraisal and Management
}

\author{
Sameh moustafa, MD, Amr M Salem, MD; * Mohamed E Salem MD.
}

\author{
Vascular Surgery Unit, Department of Surgery, Alexandria University. \\ Faculty of Medicine and Department of Surgery Medical Research Institute*
}

Background: As a result of rapidly growing population with increased incidence of DM, chronic kidney disease (CKD) and end-stage renal disease (ESRD) increased markedly worldwide. This led to increased importance of haemodialysis access creation and the care for its possible complications. In Egypt, hypertension and diabetes mellitus are important causes of ESRD with their impact on the incidence of dialysis access complications. Late complications encountered during access use in dialysis may include late thrombosis, late infection at puncture sites, aneurysm and pseudoaneurysm formation, venous hypertension and steal syndrome. Cardiopulmonary and neurologic complications may be encountered also but they were not delt with in this study.

Aim of work: To report and assess different types of complications offunctioning haemodialysis access done at Alexandria main university hospital and medical research institute in one year follow up (from January 2013 to January 2014) for late complications and their management.

Patients and methods: 160 patients with dialysis access done in the year 2012 and used for regular dialysis were included in this study. They were followed for one year, from January 2013 to January 2014 for late complications and their management aiming primarily for access salvage. Early access failure before dialysis and early infection cases were excluded from the study as well as mild neuropathy and subclinical steal. They were all subjected to clinical examination, laboratory investigations, imaging studies. Different management tools were used according to the type of complication encountered aiming primarily to life saving as well as limb and access salvage.

Results: 160 patients were included in this study. They had ESRD with haemodialysis access constructed during the year 2012 at the main Alexandria university hospital and medical research institute. They all used their access for regular dialysis and were followed for late complications during the period from January 2013 to January 2014. They were 95 males (60\%) and 65 females (40\%). 80 patients (50\%) had radiocephalic arterio-venous fistula (AVF) , 40(25\%) brachi-basilic AVF, 28(17.5\%) brachio-cephalic AVF, and 12(7.5\%) brachioaxillary shunt PTFE grafts. 43 (26.8\%) patients developed complications during the follow up period. 27 of the complicated cases (62.7\%) were infective in the form of 24(55.8\%) infected puncture site pseudoaneurysm of native fistula and 3(6.9\%) cases of infected shunt graft. They, all, resulted in access loss. The remaining complications were 9 cases of thrombosis managed by thrombectomy, 3 cases of aneurysm formation repaired, 3 cases of venous hypertension treated by ligation and by angioplasty, and one case of clinically significant steal syndrome treated by distal revascularization interval ligation operation. The non infective complications management showed a good access salvage rate (75\%) while salvage rate went down to (27.9\%) when infected cases were included.

Conclusion: The majority of complications reported in this study were infective and the limited use of prosthetic grafts minimizes this type of complications. Except for infectious complications, most of dialysis access complications can be treated and controlled or corrected with good access salvage rates. More attention and care should be given to infection control measures in dialysis units, health education and patients' hygiene and immune status as well. 


\section{Introduction:}

End stage renal disease (ESRD) is defined as the irreversible decrease in renal function, incompatible with patient life unless dialysis or transplantation is applied i.e. Renal replacement. These patients show a reduction of glomerular filtration rate (GFR) to be less than $15 \mathrm{ml} / \mathrm{min} / 1.73 \mathrm{~m}^{2}$ body surface area, or require dialysis regardless their GFR levels. ${ }^{1}$

Diabetes mellitus (DM) and hypertension, which have their own systemic general and vascular complications, are known to be the commonest causes of ESRD worldwide followed by glomerulonephritis. ${ }^{2}$ In Egyptian patients, hypertension is the leading cause of ESRD followed by DM. ${ }^{3}$ It is expected that these two systemic diseases to have their impact on the incidence of dialysis access complications.

Other rare causes of ESRD include chronic pyelonephritis, obstructive uropathy and reflux, renal neoplasms, and polycystic kidneys. $^{2}$

Due to the growing population along with increased incidence of DM, chronic kidney disease (CKD) and ESRD increased markedly to be to be 347.1 per million in the United States in $2005 .{ }^{4}$ Hence, haemodialysis access creation and the care for its possible complications are of utmost importance.

The national kidney disease outcome quality initiative (NKD-KDOQI) guidelines encourage the use of autogenous access with the emphasis of expectant management to detect access malfunction before failure. ${ }^{5}$ The idea behind encouraging autogenous access over synthetic grafts is to minimize the complications and reduce the cost of patient care as well as getting the benefit of better patency of autogenous access.

To maintain functional haemodialysis access, hospital admission and interventions are found to be significantly lower in patients having autogenous access than those with synthetic grafts. ${ }^{4,6}$

Haemodialysis access related complications can be categorized into:

- Localized complications related to the access itself including: Thrombosis, maturation failure, haemorrhage, infection, aneurysm and pseudo aneurysm formation, seroma, access-related limb ischaemia, venous hypertension, and neuropathy. ${ }^{7}$

- Cardio-pulmonary or systemic complications: Congestive heart failure ${ }^{8}$ and pulmonary hypertension. ${ }^{9}$

Late complications refer to those encountered while access is in use for dialysis they include. ${ }^{10}$

- Late access failure.

- Infection at puncture site.

- Late haemorrhage.

- Aneurysm and pseudo aneurysm formation.

- Venous hypertension.

- Steal syndrome.

Cardiopulmonary as well as neurologic complications are to be considered late complication but are not dealt with in this study which is concerned with localized vascular problems.

\section{Aim of work:}

To report and assess different types of complications of 160 haemodialysis access done at Alexandria main university hospital and medical research institute during a period of one year follow up as well as their different management strategies.

\section{Patients:}

After exclusion of early access failure and loss as well as failure of maturation, 160 patients suffering end stage renal disease having their dialysis access done in the year 2012 and used for dialysis were included in this study. They were followed for one year, from January 2013 to January 2014 for late complications and their management aiming primarily for access salvage.

The following patients were excluded from the study:

- Cases of failure of maturation.

- Early shunt thrombosis.

- Early infection with secondary haemorrhage leading to shunt loss.

- Mild neurologic complications which did not necessitate access closure.

- Subclinical steal which did not necessitate intervention. 


\section{Methods:}

The patients of this study were subjected to:

- Full history taking.

- Thorough clinical evaluation

- Lab investigations including:

- complete blood count.

- blood glucose level.

- Renal and liver function tests.

- Blood gases and electrolytes.

- $\quad$ ECG and plain chest radiogram.

- Duplex ultrasound examination of the access components: Artery and vein measuring flow across, assessing outflow venous obstruction, and distal arterial circulatory assessment whenever patient was not presented by acute bleeding.

- Angiography when: Central venous occlusive problem was suspected and in selected cases with steal.

\section{Results:}

The study included 160 patients having ESRD with haemodialysis access constructed during the year 2012 at the main Alexandria university hospital and medical research institute. All the 160 used the access for regular dialysis and were followed for late complications during the period from January 2013 to January 2014. They were 95 males $(60 \%)$ and 65 females $(40 \%)$ with their age ranged from 16 to 67 years with a mean of 48.07 \pm 9.11 SD Table (1). They had different types of haemodialysis access including: $80(50 \%)$ radiocephalic arterio- venous fistula (AVF) , 40(25\%) brachio basilic AVF, 28(17.5\%) brachio cephalic AVF, and $12(7.5 \%)$ brachio axillary shunt grafts (2ry access) Tables $(\mathbf{1 , 2})$.

$43(26.8 \%)$ patients out of 160 developed complications during one year follow up. They were 23 males $(53.5 \%)$ and 20 females (46.5\%) Table (3) with no significant age difference from the total patients studied. Their ages ranged from 18 to 65 years with a mean of $47.94 \pm 9.36 \mathrm{SD}$.

As regards sex distribution, female patients were reported to develop complications more than males $(30.7 \%$ in female patients compared to $24.2 \%$ in males).
Of the 43 reported complications, 27 $(62.7 \%)$ reported cases were due to infection. $24(55.8 \%)$ of them were in the form of infected puncture site pseudoaneurysm of native AVF while only $3(6.9 \%)$ cases of infected shunt graft were reported. All infected complications were treated by shunt ligation and/or graft removal in cases of 2ry access infection. The remaining complications were 9 cases of thrombosis, 3 cases of aneurysm formation, 3 cases of venous hypertension, and one case of clinically significant steal syndrome. Table (4).

All infective complications of native AVF presented as infected puncture site with abscess formation Figure (1), rupture and secondary haemorrhage. They were managed by shunt ligation in three radio cephalic AVF, 9 brachio basilic AVF, and 12 brachio cephalic AVF with dialysis access loss. This was done in the background of heavy infection that did not allow fistula preservation.

Three infected PTFE brachio- axillary shunt grafts were removed due to graft infection extending to graft anastomosis with late 2ry haemorrhage after a minimum of 6 months of usage in dialysis. Graft removal was done with axillary vein ligated in all cases, brachial artery ligated in two cases (with resulted compensated ischaemia of the hand), and brachial artery was repaired by a vein patch in one case where infection was not massive at the arterial anastomosis of the graft.

The nine cases of fistula thrombosis were managed by trial of thrombectomy that was successful in 5 brachio cephalic AVF and 2 brachio- basilic AVF and failed in two cases.

The brachio -cephalic AVF complicated with steal syndrome was treated by distal revascularization interval ligation (DRIL) operation with shunt salvage and improved hand ischaemia.

Three cases of brachio basilic AVF complicated with severe venous hypertension in the form of severe forearm and hand oedema along with repeated cellulitis. After medical control of cellulitis and under umbrella of antibiotics, two cases were ligated and one cases treated by balloon dilatation and 
stenting of subclavian vein stenosis.

Aneurysmal dilatation of venous limb of native AVF was encountered in three cases, one brachio basilic and two brachio cephalic AVF. Only one case presented by rupture and haemorrhage from brachio cephalic fistula. This case was treated by excision of the aneurysm with a skin ellipse, ligation of the proximal venous limb and interposition vein graft between distal venous segment and a more proximal brachial artery segment creating a new arterial anastomosis Figure (2).

The other two cases, presented by impending rupture were treated by aneurysmorrhaphy.

Dialysis access preservation was not successful in all infection cases whether 1ry or 2ry access due to heavy infection and life threatening 2ry bleeding where life saving ligation to stop bleeding was indicated.

The total access salvage rate in the study patients was only $27.9 \%$ (12 cases out of 43) due to the high prevalence of infective complication reported. However, the salvage rate is higher when excluding cases of infection i.e. $75 \%$ (12 cases salvaged out of 16) Table (6).

\section{Discussion:}

ESRD as well as dialysis access maintenance are serious health issues mounting to near the epidemic proportions in USA. ${ }^{11}$ This also applies to the problem magnitude worldwide. This is reflected on the fact that dialysis access procedures became the commonest procedure done by vascular surgeons. ${ }^{12}$ The number of this study patients (160 patients in one year), as being collected from two centers, matches with the fact that Egypt is considered one of the most 15 countries with the largest dialysis patients population. ${ }^{13}$

As regards the current study demographic data, the patients age and sex distribution matches with other studies on Egyptian patients population, ${ }^{13}$ while when compared to western studies, the Egyptian patients are younger. ${ }^{11,12}$ This is possibly due to the higher health care standards in western countries.

Due to the availability of peripheral veins suitable for AVF, and due to the general principle to avoid the use of synthetic grafts in the main Alexandria university hospital unless the patient is totally exhausted of any suitable access, the majority of the study patients had native 1ry access (92.5\%) while only $7.5 \%$ had synthetic grafts.

As most of the study patients were males, complications were more found in males. However, among female patients complications were found more i.e. $30.7 \%$ in female patients as compared to $24.2 \%$ in males. This is possibly due to better hygiene and peripheral venous status in males and/ or more prevalence of obesity in female patients which might predispose to more complications. This point needs more detailed study to be verified.

Huber et al ${ }^{14}$ reported a mortality rate of about $3 \%$ and a complication rate of $20 \%$. The current study reported no mortality possibly due to the perioperative nature of Huber et al report. Complication rate in the current study was $26.8 \%$ which was found to be higher possibly due to the higher rate of infectious complications.

The KDOQI panel stated that infectious complications should not exceed $1 \%$ for native access and 10\% for prosthetic ones during their lifetime. ${ }^{15}$ The current study reported comparable rate of native access infectious complications as the number of infected native AVF was 24 out of 148 fistulae i.e. $0.16 \%$. However, as regards the 12 prosthetic grafts followed in the current study, 3 of them were infected during the follow up period i.e. $25 \%$ which is much higher percentage when compared to KDOQI statement. As late infection, the higher incidence in the current study may be attributed to the lower level of patient hygiene or the lacking of aseptic techniques during puncture in dialysis units. Moreover, the use of button hole technique for access puncture rather than the step ladder technique may be another factor especially with the lacking of adequate post dialysis compression. These might be main causative factors as all the study infectious complications were in the form of infected puncture site. Moreover, the smaller number 


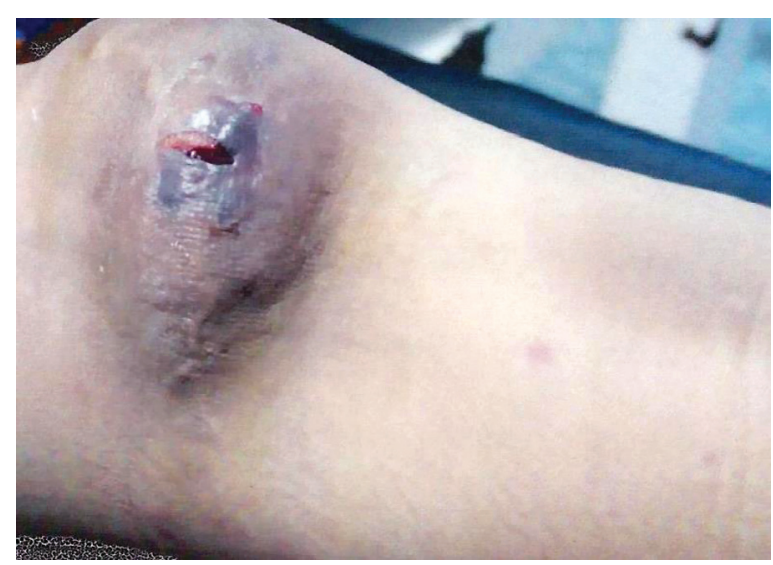

Figure (1): Infected brachiocephalic AVF with abcess formation and sealed $2 r y$ bleeding.

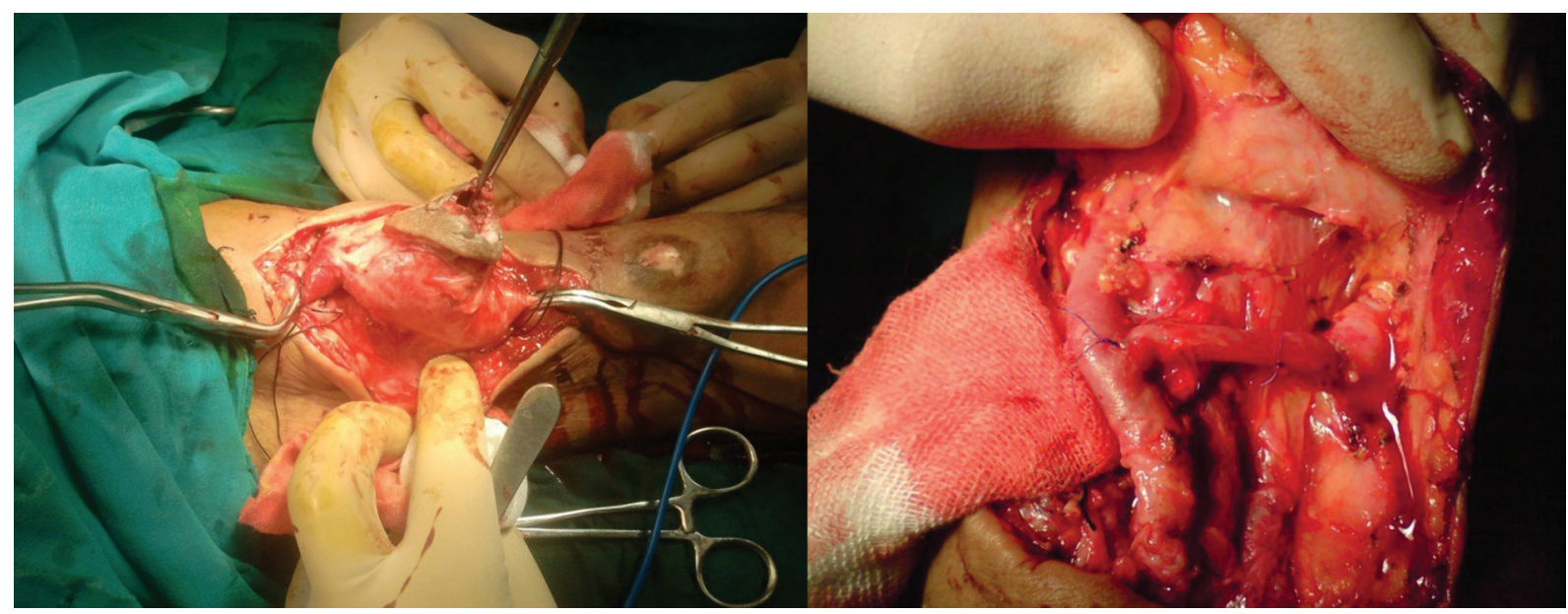

Figure (2): Ruptured AVF venous aneurysm treated by excision and brachio-cephalic interposition vein graft.

Table (1): Sex distribution of the study patients:

\begin{tabular}{|l|l|l|}
\hline Sex & Number & $\%$ \\
\hline Male & 95 & $60 \%$ \\
\hline Female & 65 & $40 \%$ \\
\hline Total & 160 & $100 \%$ \\
\hline
\end{tabular}

Table(2): Types of haemodialysis access studied :

\begin{tabular}{|l|l|l|l|}
\hline 1ry haemodialysis access & \multicolumn{3}{|l|}{ 2ry haemodialysis access } \\
\hline Type & No.(\%) & Type & No. $(\%)$ \\
\hline Radiocephalic AVF & $80(50 \%)$ & Brachio axillary & $12(7.5 \%)$ \\
PTFE shunt graft & \\
\hline Brachiobasilic AVF & $40(25 \%)$ & & \\
\hline Brachiocephalic AVF & $28(17.5 \%)$ & $12(7.5 \%)$ & \\
\hline Total & $148(92.5 \%)$ & \multicolumn{2}{|l}{} \\
\hline
\end{tabular}

of prosthetic grafts in the current study made statistical significance test impossible. So larger studies considering prosthetic grafts are required to assess this point which will require much longer time to recruit a good number of patients. This is because our institution trend is to avoid prosthetic access as much as possible. 
Table (3): Sex distribution of patients with complicated dialysis access in one year period in comparison to total study numbers:

\begin{tabular}{|l|l|l|l|}
\hline & Male & Female & Total \\
\hline Total patient number & 95 & 65 & 160 \\
\hline Number of patients with complications & 23 & 20 & 43 \\
\hline Complicated cases in comparison to total number $(\%)$ & $24.2 \%$ & $30.7 \%$ & $26.8 \%$ \\
\hline
\end{tabular}

Table (4): Types of complications in relation to their total number:

\begin{tabular}{|l|l|}
\hline \multicolumn{1}{|c|}{ Complication } & \multicolumn{1}{c|}{ Number(\%) } \\
\hline Infected native AVF & $24(55.8 \%)$ \\
\hline Infected graft & $3(6.9 \%)$ \\
\hline Thrombosis & $9(20.9 \%)$ \\
\hline Aneurysm formation & $3(6.9 \%)$ \\
\hline Venous hypertension & $3(6.9 \%)$ \\
\hline Steal syndrome & $1(2.3 \%)$ \\
\hline Total & $43(100 \%)$ \\
\hline
\end{tabular}

Table (5): Different types of complications in relation to the access type and total number of patients with functioning fistula:

\begin{tabular}{|c|c|c|c|c|c|c|c|}
\hline Access type & 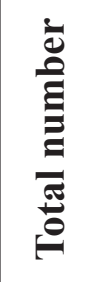 & 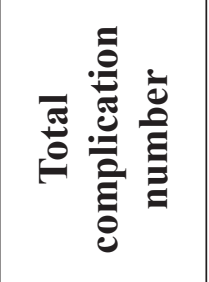 & 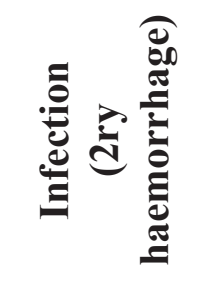 & 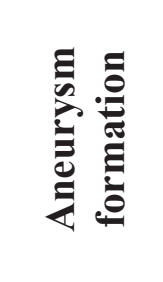 & 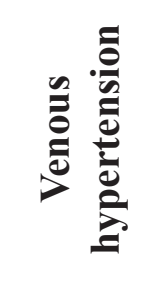 & 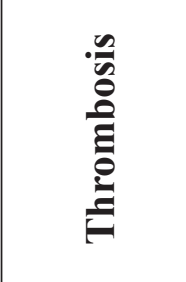 & 可 \\
\hline Radiocephalic & 80 & $4(5 \%)$ & $3(3.7 \%)$ & 0 & 0 & $1(1.3 \%)$ & 0 \\
\hline Brachiobasilic & 40 & $16(40 \%)$ & $9(22.5 \%)$ & $1(2.5 \%)$ & $3(7.5 \%)$ & $3(7.5 \%)$ & 0 \\
\hline Brachiocephalic & 28 & $20(71.4 \%)$ & $12(42.8 \%)$ & $2(7.1 \%)$ & 0 & $5(17.9 \%)$ & $1(3.6 \%)$ \\
\hline PTFE graft & 12 & $3(25 \%)$ & $3(25 \%)$ & 0 & 0 & 0 & 0 \\
\hline Total & 160 & $43(26.9 \%)$ & $27(16.9 \%)$ & $3(1.8 \%)$ & $3(1.8 \%)$ & $9(5.6 \%)$ & $1(0.6 \%)$ \\
\hline
\end{tabular}

Table (6): Access salvage rates:

\begin{tabular}{|c|c|c|c|c|c|}
\hline \multicolumn{2}{|c|}{ All complictions } & \multicolumn{2}{|c|}{ Infective complications } & \multicolumn{2}{c|}{ Non-infective complications } \\
\hline $\begin{array}{c}\text { Number of } \\
\text { complicated } \\
\text { cases }\end{array}$ & $\begin{array}{c}\text { Number of } \\
\text { preserved } \\
\text { access }\end{array}$ & $\begin{array}{c}\text { Number of } \\
\text { complicated } \\
\text { cases }\end{array}$ & $\begin{array}{c}\text { Number of } \\
\text { preserved } \\
\text { access }\end{array}$ & $\begin{array}{c}\text { Number of } \\
\text { complicated } \\
\text { cases }\end{array}$ & $\begin{array}{c}\text { Number of } \\
\text { preserved } \\
\text { access }\end{array}$ \\
\hline $43(100 \%)$ & $12(27.9 \%)$ & $27(100 \%)$ & 0 & $16(100 \%)$ & $12(75 \%)$ \\
\hline
\end{tabular}

In the current study access preservation was amenable in $75 \%$ of non-infectious complicated cases. However, the total access salvage rate was much lower i.e. $27.9 \%$. Due to the high incidence of infectious complications with access loss. This should attract the attention towards better infection control measures at dialysis units, better patient health education for better hygiene, and paying attention to the patients' immune status and general health for better results. 


\section{Conclusion:}

The main type of complications of haemodialysis access we reported in this study was infectious complications.

- It is more observed in prosthetic types of access.

- The wise limited use of synthetic grafts in our patients was found to limit complications especially of infective aetiology.

- Except for infectious complications, most of dialysis access complications can be treated and controlled or corrected with good access salvage rates.

- More attention and care should be given to infection control measures, health education and patients' hygiene as well.

- Dialysis units nursing staff education and adequate training should be promoted concerning these issues with multidisciplinary team approach including nephrologists, nursing staff and vascular surgeons.

\section{Reference:}

1- KDOQI: National Kidney Foundation. II. Clinical practice guidelines and clinical practice recommendations for anemia in chronic kidney disease in adults. $A m J$ Kidney Dis 2006; 47(5,3): 16-85.

2- Abboud H, Henrich WL: Clinical practice. Stage IV chronic kidney disease. $N$ Engl $J$ Med 2010; 362: 56-65.

3- The Egyptian Renal Registry. $9^{\text {th }}$ Annual Report. Adel Afifi, MD. Professor of Internal Medicine \& Nephrology 2008.

4- NKF-DOQI: Clinical practice guidelines for vascular access. National Kidney FoundationDialysis Outcomes Quality Initiative. Am J Kidney Diseasae 1997; 30: 150-191.

5- NKF-DOQI: Clinical practice guidelines for vascular access. Am J Kidney Disease 2001; 37: 137-181.
6- Lok CE, Oliver MJ: Overcoming barriers to arteriovenous fistula creation and use. SeminDial 2003; 16:189-196.

7- Sidawy AN, Gray R, Besarab A, et al: Recommended standards for reports dealing with arteriovenous hemodialysis accesses. $J$ Vasc Surg 2002; 35: 603-610.

8- Wijnin E, et al: The relation between vascular access flow and different types of vascular access with systemic hemodynamics in hemodialysis patients. Artif Organs 2005; 29: 960-964.

9- Carmine Zoccali: Pulmonary hypertension in dialysis patients: A prevalent, risky but still uncharacterized disorder. Nephrol Dial Transplant 2012; 27: 3674-3677.

10- Lazaredes MK, Georgiadis GS, Antaniou GA, et al: A meta-analysis of dialysis access outcome in elderly patients. J Vasc Surg 2007; 45: 420-426.

11- U.S. Renal Data System: USRDS 2012 annual data report: Atlas of end-stage renal disease in the United States, Bethesda, MD, 2012, National Institutes of Health, National Institute of Diabetes and Digestive and Kidney Diseases.

12- Woo K, Farber A, Doros G, et al: Evaluation of the efficacy of the transposed upper arm arteriovenous fistula: A single institutional review of 190 basilic and cephalic vein transposition procedures. J Vasc Surg 2007; 46: 94-99.

13- Soliman AR, Fathy A, Roshd D: The growing burden of end-stage renal disease in Egypt 2012; 34(4): 425-428.

14- Huber TS, Ozakie CK, Flynn TC, et al: Prospective validation of an algorithm to maximize native arteriovenous fistulae for chronic hemodialysis access. $J$ Vasc Surg 2002; 36: 452-459.

15- National Kidney Foundation: K/DOQI Clinical Practice Guidelines for Vascular Access, 2006 updates. Am J Kidney Dis 2001; 37: 137-181. 


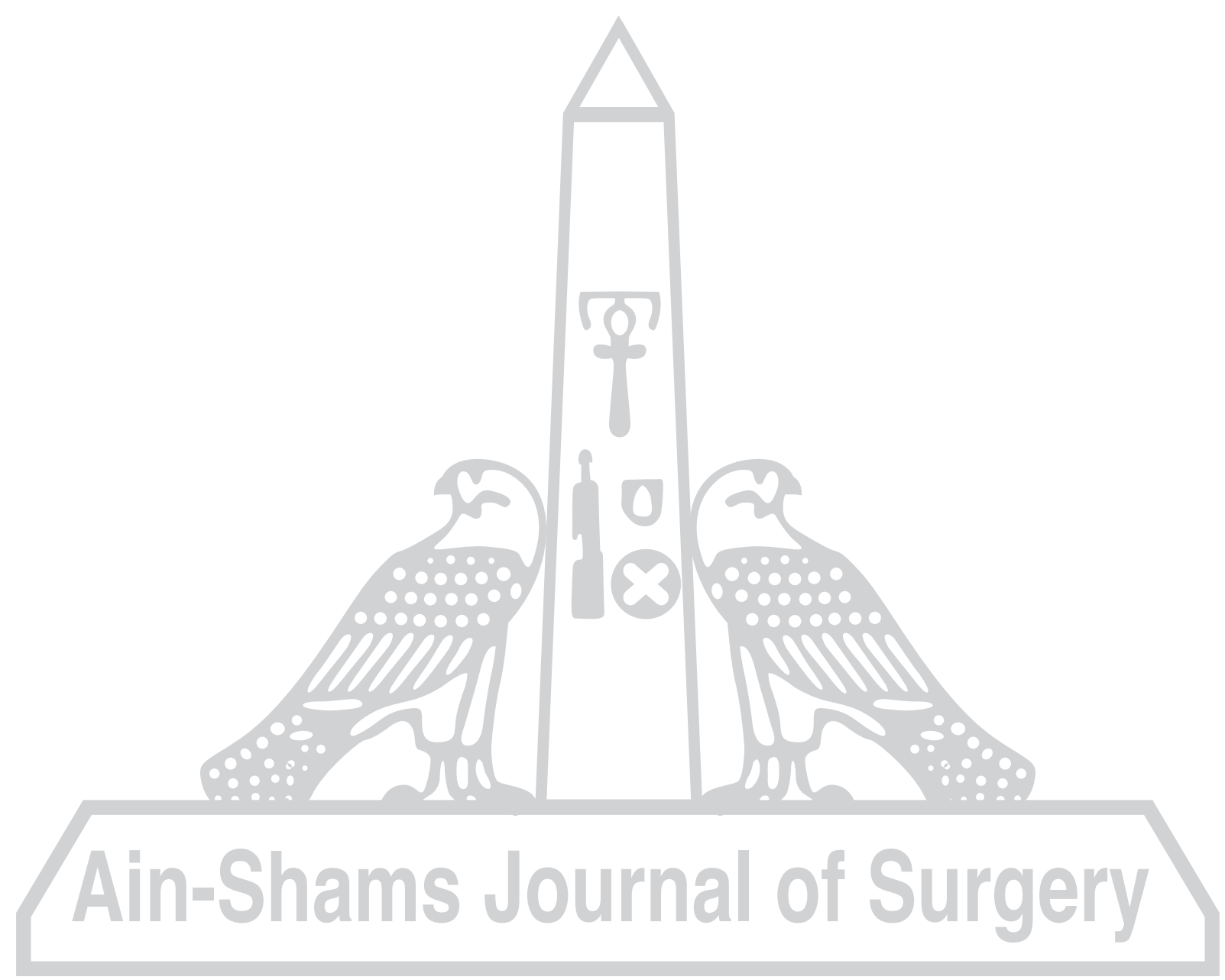

